\title{
Evaluation of circulating cell-free nuclear and mitochondrial DNA levels in Syrian patients with breast tumor
}

\author{
MILDA SAFI $^{1}$ and ABDUL RAHMAN NAJIB ${ }^{2}$ \\ ${ }^{1}$ Department of Biochemistry and Microbiology, Faculty of Pharmacy, Damascus University; \\ ${ }^{2}$ Department of Biostatistics, Faculty of Statistics, Damascus University, Damascus 22743, Syria
}

Received January 23, 2020; Accepted September 3, 2020

DOI: $10.3892 /$ etm.2020.9497

\begin{abstract}
In the last decade, the roles of circulating cell free nuclear (ccfn) and ccf mitochondrial (ccfmt) DNA as potential noninvasive biomarkers have been demonstrated in numerous different types of disease, including cancer. However, the results remain controversial. The present study aimed to investigate the roles of ccfnDNA and ccfmtDNA levels in the plasma of patients with breast cancer. A total of 84 Syrian female subjects were included in the study, who were divided into 3 groups: i) Malignant disease group ( $n=33)$; ii) benign disease group $(n=26)$; and iii) healthy control group $(n=25)$. CcfnDNA and ccfmtDNA were determined using real-time quantitative PCR and the reactions were followed by melting curve analysis. The results indicated no significant differences in the plasma levels of ccfnDNA, ccfmtDNA or the ratio of ccfmtDNA/ccfnDNA between the study groups. Of note, a positive correlation was observed between the ccfmtDNA/ccfnDNA ratio and age in the control group $(\mathrm{P}=0.012 ; \mathrm{r}=0.505)$. In addition, a positive correlation was identified between ccfnDNA levels and the estrogen receptor status $(\mathrm{P}=0.045 ; \mathrm{r}=0.416)$, while a negative correlation between ccfmtDNA/ccfnDNA ratio and the progesterone receptor status was obtained $(\mathrm{P}=0.045 ; \mathrm{r}=-0.448$. Aging and the role of hormones in the cells may be responsible for these results. In the future, the present study should be followed up with mutation detection analyses and large-scale studies.
\end{abstract}

\section{Introduction}

Breast cancer is one of the most common malignancies, accounting for $29 \%$ of all cancer cases in females worldwide (1). In the Middle East, the prevalence of breast cancer remains lower than that in North America and Europe, where it accounts for $14-42 \%$ of all female cancer cases (2). In Syria, there are

Correspondence to: Dr Milda Safi, Department of Biochemistry and Microbiology, Faculty of Pharmacy, Damascus University, 15 Almazza Highway, Damascus 22743, Syria

E-mail: milda_2002@hotmail.com

Key words: circulating cell-free mitochondrial DNA, circulating cell-free nuclear DNA, breast cancer, plasma, Syria no official statistics on the prevalence of breast cancer or other malignancies (3). According to the International Agency for Research on Cancer in 2012, the prevalence ratio of breast cancer in females in Syria is $36.4 \%$ and the mortality rate is $24.8 \%$ (4). In recent years, numerous methods have been used for screening, diagnosing and monitoring breast cancer, including physical examination, mammography and biopsy (5). However, each of these techniques has numerous drawbacks, making it necessary to identify a novel diagnostic biomarker or method (6). In addition, despite the significant progress in the development of cancer therapies, cancer-associated mortality remains high (7). Previous research has increasingly focused on the role of the quantitative and qualitative changes of circulating cell-free nuclear DNA (ccfnDNA) and circulating cell-free mitochondrial DNA (ccfmtDNA) in numerous different types of disease, but particularly in cancer $(8,9)$. Cancer cells release their nucleic acids (both $\mathrm{n} / \mathrm{mDNA}$ and RNAs) into the systemic circulation through cellular necrosis and apoptosis $(10,11)$; this DNA is mixed with DNA released from normal cells (12). In previous studies, changes in the ccfnDNA and ccfmtDNA levels were identified not only in tissue samples but also in body fluids such as plasma, which makes them attractive noninvasive potential biomarkers $(13,14)$. Several studies have reported elevated levels of ccfnDNA or ccfmtDNA in different types of cancer, while others reported decreased levels, thus the results remain conflicting (15-17). Due to these results, the present study aimed to determine their role in breast cancer by comparing the absolute quantities of ccfnDNA and ccfmtDNA, as well as the ccfmtDNA/ccfnDNA ratio, among malignant and benign tumor groups and healthy controls.

\section{Materials and methods}

Patients. In total, 84 Syrian females were included in the present study. Informed consent forms were signed by all participants and the study was approved by the Syrian Higher Commission for Scientific Research, Ministry of Higher Education, Damascus, Syria. The study cohort $(n=84)$ was divided into 3 groups: i) Malignant disease group $(n=33)$; ii) benign disease group $(n=26)$; and iii) healthy control group $(n=25)$. Groups 1 and 2 contained patients encountered at the general surgery department of Al-Assad University Hospital-Damascus (Damascus, Syria) for breast mastectomy; the diagnosis was confirmed by biopsy for all cases. The healthy control group used in this study had neither a history of cancer nor suffered from any other severe or autoimmune 
disease. All blood samples were taken prior to any invasive procedures or therapeutic treatments between February 2013 and June 2014. The clinical data of each patient [age, smoking status, menopausal status, cancer stage, grade, tumor size, lymph node involvement, estrogen receptor (ER), progesterone receptor (PR) and human epidermal growth factor receptor (Her2-neu) status] were obtained from the pathological reports (Table I). The blood samples $(2 \mathrm{ml})$ were centrifuged twice, first at $1,600 \mathrm{x}$ g for $10 \mathrm{~min}$ at $4^{\circ} \mathrm{C}$ and then at $16,000 \mathrm{x}$ g for $10 \mathrm{~min}$ at $4^{\circ} \mathrm{C}$ (each time with the Centrifuge 5417R; Eppendorf). The supernatant was stored at $-80^{\circ} \mathrm{C}$ until the next step. The DNA was extracted using the GeneJETGenomic DNA purification kit (Thermo Fisher Scientific, Inc.) according to the manufacturer's protocol, except for a change of the elution buffer volume from 200 to $100 \mu \mathrm{l}$. DNA extracts were stored at $-80^{\circ} \mathrm{C}$ until further use. CcfnDNA and ccfmtDNA levels were measured via real-time quantitative PCR using a LightCycler instrument (Roche Diagnostics GmbH). One primer pair specific for the nDNA $(\beta 2 \mathrm{M})$ and another primer pair for the mDNA (D-loop) were used for quantification (TIB Molbiol). The primers pairs were selected after reviewing a large number of scientific articles (15) and their specificity was analyzed using BLAST (https://blast.ncbi.nlm.nih.gov/; date of accession April 25th, 2019) and Primer BLAST (https://www.ncbi.nlm. nih.gov/tools/primer-blast/; date of accession April 25th, 2019). The primer sequences are listed in Table II. Standard curves for nDNA and mtDNA were generated using homemade synthetic target amplicons. The standards were synthesized through DNA isolation, conventional PCR, PCR product purification, DNA sequencing, measuring the concentration and finally confirming that the sample and the standard were amplified with identical efficiencies. Each sample was assayed in duplicate using FastStart DNA Master SYBR Green 1 (Roche Diagnostics GmbH) using a different primer pair each time. qPCR was performed in a total reaction volume of $20 \mu \mathrm{l}$, containing $3 \mathrm{mM} \mathrm{MgCl}_{2}, 0.5 \mu \mathrm{M}$ forward primer and $0.5 \mu \mathrm{M}$ reverse primer. The following thermocycling conditions were used: Initial denaturation at $95^{\circ} \mathrm{C}$ for $10 \mathrm{~min}$, followed by 55 cycles of denaturation at $95^{\circ} \mathrm{C}$ for $10 \mathrm{sec}$, annealing at $60^{\circ} \mathrm{C}$ for $10 \mathrm{sec}$ and elongation at $72^{\circ} \mathrm{C}$ for $7 \mathrm{sec}(18)$. All of the reactions were followed by melting curve analysis. The copy numbers per $\mathrm{ml}$ of plasma for ccfnDNA and ccfmtDNA in samples were quantified using the following equation:

$$
\text { Qnuclear }=\frac{\mathrm{C}}{3.3} \times \frac{\text { Velution }}{\text { Vplasma }}
$$

Qnuclear is the ccfnDNA copy number per milliliter, $\mathrm{C}$ is the ccfnDNA concentration $(\mathrm{pg} / \mu \mathrm{l})$ determined by qPCR targeting the $\beta 2 \mathrm{M}$ gene sequence and $3.3 \mathrm{pg}$ is the human haploid genome mass. $V_{\text {elution }}$ is the volume of ccfDNA extract $(\mu l)$ and $\mathrm{V}_{\text {plasma }}$ is the volume of plasma used for the extraction (ml).

$$
\text { Qmitochondrial }=\frac{\mathrm{C} \times \mathrm{Na}}{2 \times \mathrm{MW} \times \mathrm{Ln}} \times \frac{\text { Velution }}{\text { Vplasma }}
$$

$\mathrm{Q}_{\text {mitochondrial }}$ is the ccfmtDNA copy number per milliliter and $\mathrm{C}$ is the ccfmtDNA concentration (pg/ $\mu \mathrm{l})$ determined by a qPCR targeting the mitochondrial D-loop. Na is Avogadro's number $\left(6.02 \times 10^{23}\right.$ molecules per mole), $\mathrm{Ln}$ is the nucleotide length and $\mathrm{MW}$ is the molecular weight of one nucleotide $(\mathrm{g} / \mathrm{mol}) . \mathrm{V}_{\text {elution }}$ is the elution volume of ccfDNA extract $(\mu l)$ and $V_{\text {plasma }}$ is the volume of plasma used for the extraction $(\mathrm{ml})$.

Statistical analysis. All statistical analyses were performed using SPSS statistics 13.0 software (SPSS, Inc.). Statistical differences between ccfnDNA or ccfmtDNA concentrations and the ccfmtDNA/ccfnDNA ratio were compared using ANOVA followed by Bonferroni's post-hoc test. The Kolmogorov-Smirnov test confirmed that the data were normally distributed. The correlation of the mean values with other parameters was assessed by calculating Pearson's and Spearman's correlation coefficients. Student's t-test was used to determine the association of ccfnDNA, ccfmtDNA or the ccfnDNA/ccfmtDNA ratio with menopause, smoking and lymph node status, while Pearson's correlation was used to study the correlation between ccfnDNA, ccfmtDNA and ccfnDNA/ccfmtDNA levels with age and tumor size. Furthermore, Spearman's correlation analysis was used to study the correlation between ccfnDNA, ccfmtDNA and ccfnDNA/ccfmtDNA levels with the tumor stage or grade, or with the ER, PR and Her2/neu receptor status. $\mathrm{P}<0.05$ was considered to indicate statistical significance.

\section{Results}

Plasma levels of ccfnDNA, ccfmtDNA and ccfnDNA/ccfmtDNA between the study groups. The mean ccfnDNA concentration in the breast cancer, benign tumor and the control group was $6,448,6,197$ and 6,071 copies/ml respectively, while the mean value of the ccfmtDNA concentration was $20,770,515$, $6,572,829$ and 5,188,000 copies/ml, respectively. The mean value of the ccfmtDNA/ccfnDNA ratio was 154,017, 2,155 and 24,192 in the breast cancer, benign tumor and control group, respectively. When comparing the plasma levels of ccfnDNA and ccfmtDNA and the ccfmtDNA/ccfnDNA ratio between the three study groups using ANOVA, no significant differences were identified between the groups $(\mathrm{P}=0.979,0.542$ and 0.447 , respectively; $\mathrm{P}>0.05$ ) data not shown.

Correlation between ccfnDNA, ccfmtDNA or ccfmtDNA/ccfnDNA levels and demographic features (age, menopause and smoking status). The mean age for females with breast cancer, benign tumor and control subjects was 46 (range, 28-90), 37 (range, 21-75) and 43 (range, 24-76) years, respectively. When calculating Pearson's correlation coefficients for ccfnDNA or ccfmtDNA levels or the ccfmtDNA/ccfnDNA ratio with age, menopause or smoking status in the three study groups, a positive correlation was only identified between the ccfmtDNA/ccfnDNA ratio and age in the control group $(\mathrm{P}=0.012, \mathrm{r}=0.505$; Fig. 1$)$. However, in the three study groups, no statistical significance was obtained for the correlation between the levels of ccfnDNA or ccfmtDNA or the ccmtDNA/ccfnDNA ratio and demographic features (data not shown; $\mathrm{P}>0.05$ ).

Correlation between ccfnDNA, ccfmtDNA or ccfmtDNA/ccfnDNA levels in breast cancer and the clinicopathological parameters (stage, grade, lymph node involvement, tumor size, ER, PR and Her2/neu receptor status). In the breast cancer group, no statistically significant correlations between the levels of ccfnDNA or ccfmtDNA or the ccfnDNA/ccfmtDNA ratio and the stage, grade, lymph node status or tumor size were obtained $(\mathrm{P}>0.05)$. When calculating the Spearman's correlation coefficient for the correlation between ccfnDNA or ccfmtDNA or the ccfmtDNA/ccfnDNA ratio with the ER, PR 
Table I. Parameters of the study cohort.

\begin{tabular}{|c|c|c|c|c|c|c|}
\hline \multirow[b]{2}{*}{ Group } & \multirow[b]{2}{*}{$\mathrm{N}$} & \multirow[b]{2}{*}{ Age (years) } & \multicolumn{2}{|c|}{ Smoking status (\%) } & \multicolumn{2}{|c|}{ Menopausal status (\%) } \\
\hline & & & Smoking & Non-smoker & Premenopause & Postmenopause \\
\hline Malignant disease & 33 & $46 \pm 12.8$ & 30 & 70 & 70 & 30 \\
\hline Benign disease & 26 & $37 \pm 13.6$ & 19 & 81 & 89 & 11 \\
\hline Healthy control & 25 & $43 \pm 13.6$ & 28 & 72 & 86 & 32 \\
\hline
\end{tabular}

Age is expressed as the mean \pm standard deviation.

Table II. Primer sequences used for PCR.

\begin{tabular}{llll}
\hline Gene ID & Primer name & \multicolumn{1}{c}{ Sequence $\left(5^{\prime}-3^{\prime}\right)$} & Amplicon length \\
\hline$\beta 2 \mathrm{M}$ & h $\beta 2 \mathrm{MF} 2$ & GCTGGGTAGCTCTAAACAATGTATTCA & 94 \\
& h $\beta 2 \mathrm{MR} 2$ & CCATGTACTAACAAATGTCTAAAATGGT & 64 \\
D-loop & hmitoF5 & CTTCTGGCCACAGCACTTAAAC & \\
& hmitoR5 & GCTGGTGTTAGGGTTCTTTGTTTT & \\
\hline
\end{tabular}

F, forward; R, reverse.

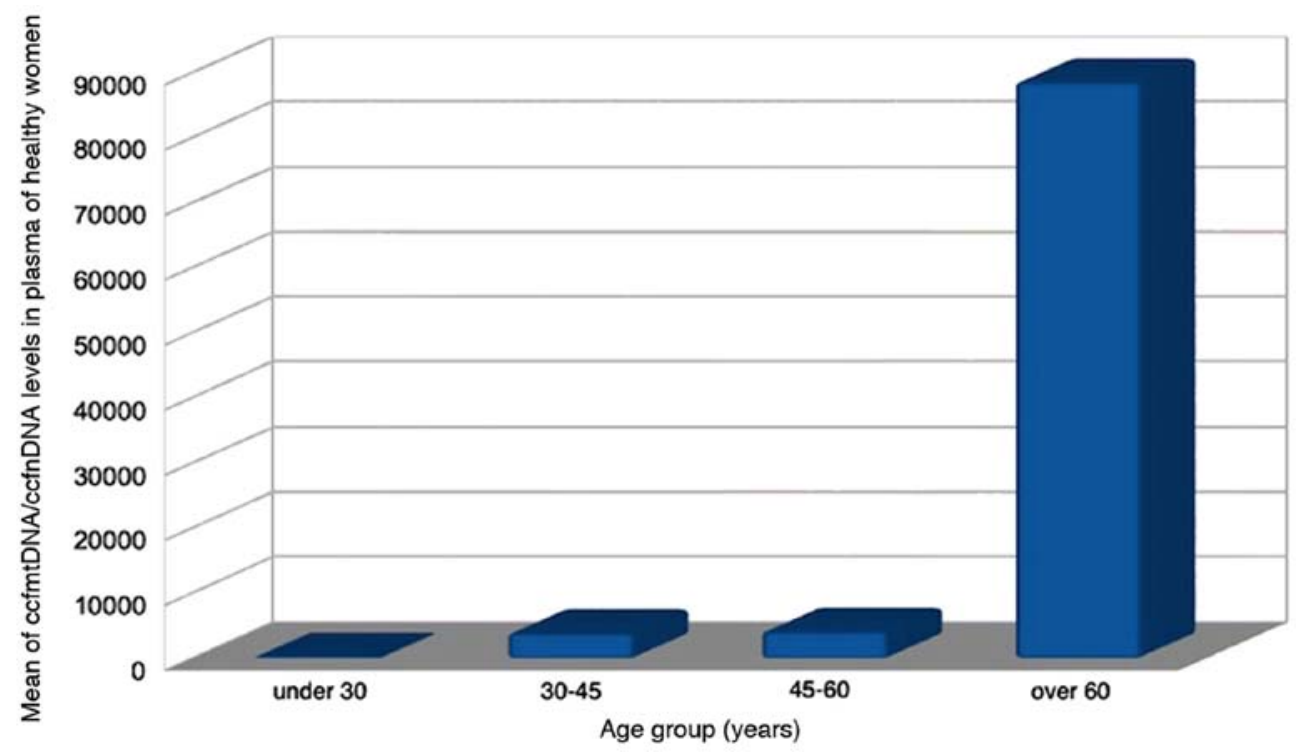

Figure 1. Association between mean values of ccfmtDNA/ccfnDNA levels in the plasma of healthy females with age. After dividing the females into four groups based on age (x-axis), a significant increase was found in the levels of ccfmtDNA/ccfnDNA (y-axis) with increasing age especially in the over 60 group. ccfmtDNA, circulating cell-free mitochondrial DNA; ccfnDNA, circulating cell-free nuclear DNA.

or Her2/neu receptors status, a positive correlation was identified between ccfnDNA levels and ER status $(\mathrm{P}=0.045, \mathrm{r}=0.416$; Fig. 2A), while a negative correlation was obtained between the ccfmtDNA/ccfnDNA ratio and PR status $(\mathrm{P}=0.045 ; \mathrm{r}=-0.478$; Fig. 2B and Table III).

\section{Discussion}

For breast cancer in general, but particularly in the Middle East, there remains a lack of research in the field of ccfDNA, and it is required to further investigate its role. In the present study, PCR with SYBR-Green I was used for the quantification and numerous previous studies were reviewed to ensure that the most specific primers for both nDNA and mDNA were used, and their specificity was tested prior to using them. Each PCR was also followed up by melting curve analysis to ensure the accuracy of the results. Neither the concentrations of ccfnDNA and ccfmtDNA, nor the ccfmtDNA/ccfnDNA ratio were significantly different between the three study groups, which differs from the results of other previous studies (15-17). This difference may be explained through ethnic differences, limited sample sizes and differences 

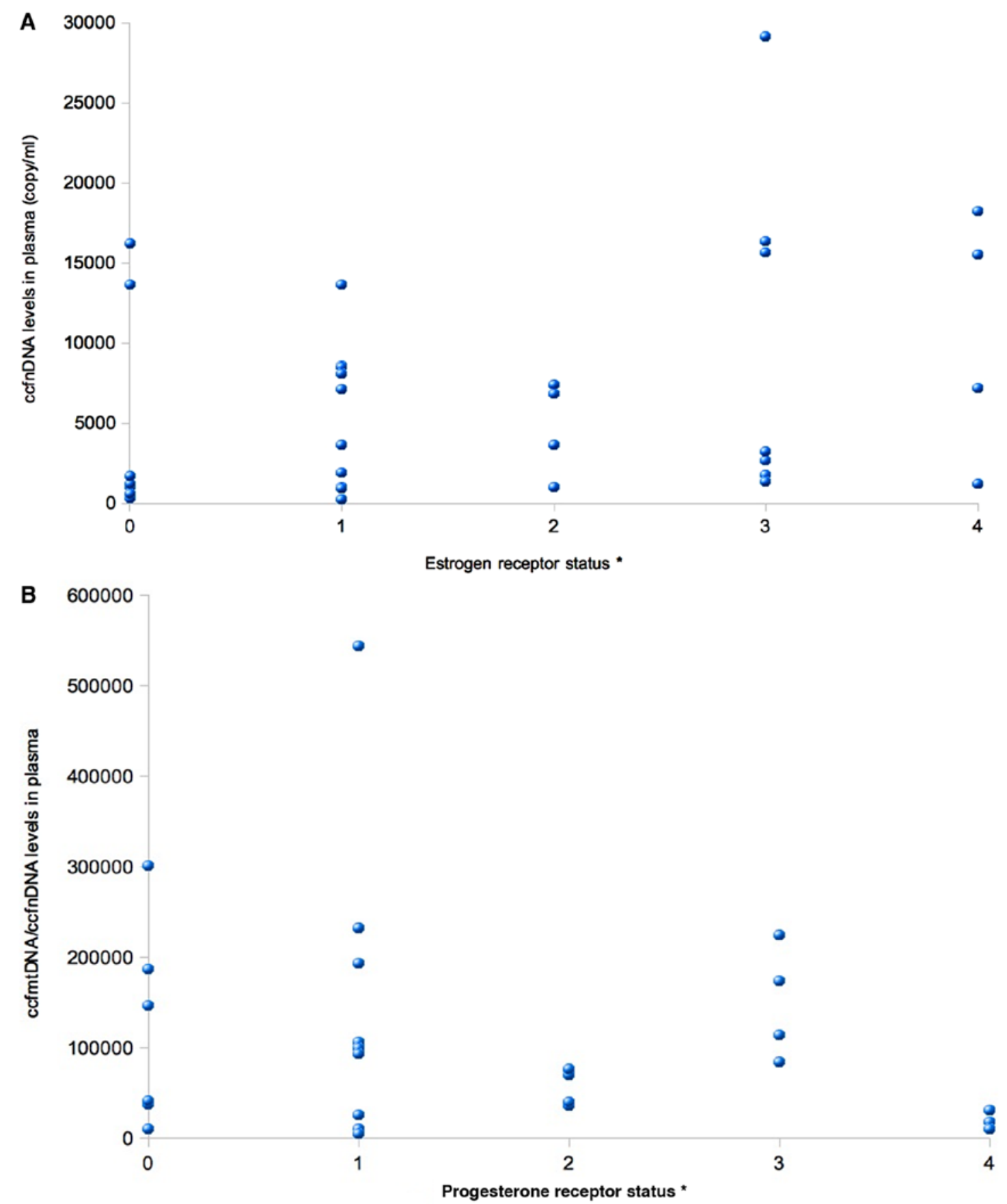

Figure 2. Scatterplots for correlation of plasma levels of ccfnDNA with ER status and ccfmtDNA/ccfnDNA levels with progesterone receptor status in patients with breast cancer. (A) After dividing patients with breast cancer into five groups based on the expression status of ER in tumor cells (x-axis), a positive correlation was found between ccfnDNA levels in plasma ( $\mathrm{y}$-axis) with ER status $(\mathrm{P}=0.045 ; \mathrm{r}=0.416)$. (B) After dividing patients with breast cancer into five groups based on the expression status of PR in tumor cells (x-axis), a negative correlation was found between ccfmtDNA/ccfnDNA levels (y-axis) with PR status $(\mathrm{P}=0.045 ; \mathrm{r}=-0.448)$. Spearman's correlation analysis was performed on the datasets. 0 indicates no expression, while the increasing numbers indicate the increasing receptor expression intensity in tumor cells. ER, estrogen receptor; PR, progesterone receptor; ccfmtDNA, circulating cell-free mitochondrial DNA; ccfnDNA, circulating cell-free nuclear DNA.

in the methods used for quantification. Furthermore, the results of the present study indicated no correlations between ccfnDNA, ccfmtDNA or ccfmtDNA/ccfnDNA levels and demographic features or clinicopathological parameters, which is consistent with the results of other previous studies, except that the present study reported a positive correlation between the ccfmtDNA/ccfnDNA ratio and age in the healthy control group. This result may be due to increased levels of ccfmDNA, decreased levels of ccfnDNA or the impaired interaction between mtDNA and nDNA. In addition, it may be explained through the increased levels of reactive oxygen species with aging, which is directly responsible for damaging cells and releasing their nDNA and mtDNA into the blood stream (19) or increasing the mutation rate for both nDNA and mtDNA, but with much higher rates for mtDNA $(20,21)$. On the other hand, due to the complex relationship between mtDNA and nDNA, mutations in mtDNA may affect the way mtDNA and nDNA communicate with each other (22). The 


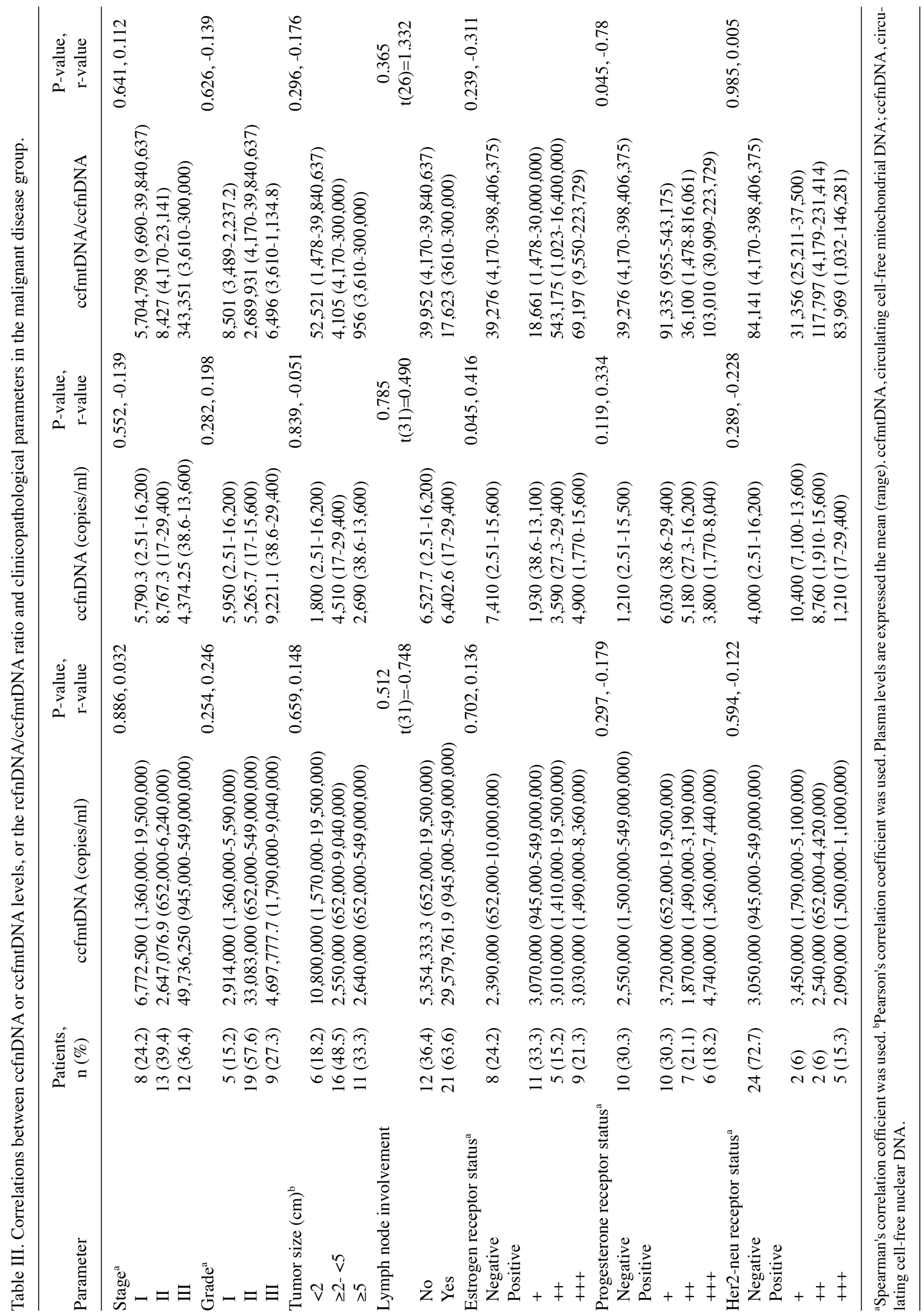


present study also revealed a positive correlation between ccfnDNA levels and ER status, which may be linked to the role of ER signaling in downregulating the DNA damage and repair pathway in mammary tissues (23). In healthy tissues, ER activity was discovered to be governed by highly regulated genetics that keep ER activity under control (24). Furthermore, dysregulated ER signals were indicated to lead to increased proliferation, the accumulation of DNA damage and eventually tumorigenesis $(23,25)$. This increase in the rate of proliferation was reported to lead to increased levels of ccfnDNA in the plasma of patients with breast cancer (17). On the other hand, a negative correlation was identified between the ccfmtDNA/ccfnDNA ratio and the PR status. Similar to ER-positive cells, PR-positive cells demonstrate loss of or alterations in the DNA damage and repair pathway or checkpoint controlling system, and an increased proliferation rate, eventually leading to cancer formation (26). Of note, all previous research in this field has reported positive trends in these particular parameters, which is promising (15-17); however, the conflicting results should be followed up with large-scale studies and a mutation analysis to clarify the relationship between ccfmtDNA, ccfnDNA and breast cancer.

In conclusion, a positive correlation was identified between ccfmtDNA/ccfnDNA levels and age in healthy individuals. In addition, a positive correlation was observed between ccfnDNA and the ER status, while a negative correlation was observed between ccfmtDNA/ccfnDNA levels and the PR status. Despite the small sample size, these results suggested that the plasma levels of ccfmtDNA and ccfnDNA may serve as a non-invasive biomarkers; however, large-scale studies are required to validate the results and clarify the conflicting results.

\section{Acknowledgements}

The authors would like to thank Professor Dr Fawza Monem and Dr Wafa Habbal from the Laboratories Department for facilitating the work in the laboratories of Al-Asaad Hospital (Damascus, Syria).

\section{Funding}

The present study was funded by the Scientific Research Office, Damascus University.

\section{Availability of data and materials}

All data generated or analyzed during this study are included in this published article.

\section{Authors' contributions}

MS designed the study and performed the molecular biology studies. MS and ARN analyzed the data; MS drafted the manuscript and ARN revised it. All authors read and approved the final manuscript.

\section{Ethics approval and consent to participate}

Informed consent forms were signed by all participants and the study was approved by the Syrian Higher Commission for
Scientific Research, Ministry of Higher Education, Damascus, Syria.

\section{Patient consent for publication}

Not applicable.

\section{Competing interests}

The authors declare that they have no competing interests.

\section{References}

1. Luffarelli P, Manna E and Fortunato L: Epidemiology and risk factors. In: Ductal carcinoma in situ of the breast. Mariotti C (ed). Springer, Cham, pp23-28, 2017.

2. Chouchane L, Boussen H and Sastry KS: Breast cancer in Arab populations: Molecular characteristics and disease management implications. Lancet Oncol 14: e417-e424, 2013.

3. El Saghir NS and Abulkhair O: Epidemiology prevention and management guidelines for breast cancer in Arab countries. Pan Arab J Oncol 3: 12-18, 2010.

4. World Health Organization: Estimated Cancer Incidence, Mortality and Prevelence Worldwide in 2012. Available from http://globocan.iarc.fr/Pages/fact_sheets_population. aspx? country $=818$. Accessed 30 October, 2017.

5. Marmot MG, Altman DG, Cameron DA, Dewar JA, Thompson SG and Wilcox M: The benefits and harms of breast cancer screening: An independent review. Br J Cancer 108: 2205-2240, 2013.

6. Freimanis RI and Yacobozzi M: Breast cancer screening. NC Med J 75: 117-120, 2014.

7. Alix-Panabières $\mathrm{C}$, Schwarzenbach $\mathrm{H}$ and Pantel $\mathrm{K}$ : Circulating tumor cells and circulating tumor DNA. Annu Rev Med 63: 199-215, 2012.

8. Elshimali YI, Khaddour H, Sarkissyan M, Wu Y and Vadgama JV: The clinical utilization of circulating cell free DNA (CCFDNA) in blood of cancer patients. Int J Mol Sci 14: 18925-18958, 2013.

9. Vymetalkova V, Cervena K, Bartu L and Vodicka P: Circulating cell-free DNA and colorectal cancer: A systematic review. Int J Mol Sci 19: 3356, 2018.

10. Yu M, Stott S, Toner M, Maheswaran S and Haber DA: Circulating tumor cells: Approaches to isolation and characterization. J Cell Biol 192: 373-382, 2011.

11. Zhou Q, Li W, Leng B, Zheng W, He Z, Zuo M and Chen A: Circulating cell free DNA as the diagnostic marker for ovarian cancer: A systematic review and meta-analysis. PLoS One 11: e0155495, 2016.

12. Rykova EY, Morozkin ES, Ponomaryova AA, Loseva EM, Zaporozhchenko IA, Cherdyntseva NV, Vlassov VV and Laktionov PP: Cell-free and cell-bound circulating nucleic acid complexes: Mechanisms of generation, concentration and content. Expert Opin Biol Ther 12 (Suppl 1): S141-S153, 2012.

13. Ignatiadis M, Lee M and Jeffrey SS: Circulating tumor cells and circulating tumor DNA: Challenges and opportunities on the path to clinical utility. Clin Cancer Res 21: 4786-4800, 2015.

14. Schwarzenbach H, Hoon DS and Pantel K: Cell-free nucleic acids as biomarkers in cancer patients. Nat Rev Cancer 11: 426-437, 2011.

15. Malik AN, Shahni R, Rodriguez-de-Ledesma A, Laftah A and Cunningham P: Mitochondrial DNA as a non-invasive biomarker: Accurate quantification using real time quantitative PCR without co-amplification of pseudogenes and dilution bias. Biochem Biophys Res Commun 412: 1-7,2011.

16. Kohler C, Radpour R, Barekati Z, Asadollahi R, Bitzer J, Wight E, Bürki N, Diesch C, Holzgreve W and Zhong XY: Levels of plasma circulating cell free nuclear and mitochondrial DNA as potential biomarkers for breast tumors. Mol Cancer 8: 105, 2009.

17. Meddeb R, Dache ZAA, Thezenas S, Otandault A, Tanos R, Pastor B, Sanchez C, Azzi J, Tousch G, Azan S, et al: Quantifying circulating cell-free DNA in humans. Sci Rep 9: 5220, 2019.

18. Springer J, Loeffler J, Heinz W, Schlossnagel H, Lehmann M, Morton O, Rogers TR, Schmitt C, Frosch M, Einsele H and Kurzai O: Pathogen-specific DNA enrichment does not increase sensitivity of PCR for diagnosis of invasive aspergillosis in neutropenic patients. J Clin Microbiol 49: 1267-1273, 2011. 
19. Sena LA and Chandel NS: Physiological roles of mitochondrial reactive oxygen species. Mol Cell 48: 158-167, 2012.

20. Desler C, Marcker ML, Singh KK and Rasmussen LJ: The importance of mitochondrial DNA in aging and cancer. J Aging Res 2011: 407536, 2011

21. Veitia R, Govindaraju D, Bottani S and Birchler J: Aging: Somatic mutations, epigenetic drift and gene dosage imbalance. Trends Cell Biol 27: 299-310, 2017.

22. Doynova MD, Berretta A, Jones MB, Jasoni CL, Vickers MH and O'Sullivan JM: Interactions between mitochondrial and nuclear DNA in mammalian cells are non-random. Mitochondrion 30: 187-196, 2016

23. Matta J, Morales L, Ortiz C, Adams D, Vargas W, Casbas P, Dutil J, Echenique M and Suárez E: Estrogen receptor expression is associated with DNA repair capacity in breast cancer. PLoS One 11: e0152422, 2016.
24. Caldon CE: Estrogen signaling and the DNA damage response in hormone dependent breast cancers. Front Oncol 4: 106, 2014.

25. Finlay-Schultz J, Gillen AE, Brechbuhl HM, Ivie JJ, Matthews SB, Jacobsen BM, Bentley DL, Kabos P and Sartorius CA: Breast cancer suppression by progesterone receptors is mediated by their modulation of estrogen receptors and RNA polymerase III. Cancer Res 77: 4934-4946, 2017.

26. Liou GY and Storz P: Reactive oxygen species in cancer. Free Radic Res 44: 479-496, 2010.

(i) This work is licensed under a Creative Commons Attribution-NonCommercial-NoDerivatives 4.0 International (CC BY-NC-ND 4.0) License. 